\title{
No association of polymorphisms in the gene encoding 5-lipoxygenase-activating protein and myocardial infarction in a large central European population
}

Werner Koch, PhD ${ }^{1}$, Petra Hoppmann, MD ${ }^{1}$, Jakob C. Mueller, PhD' ${ }^{2}$, Albert Schömig, $M D^{1}$, and Adnan Kastrati, MD ${ }^{1}$

\begin{abstract}
Purpose: Haplotypes based on polymorphisms in the gene encoding 5-lipoxygenase-activating protein have been linked with susceptibility to myocardial infarction in Iceland and the United Kingdom. We sought to replicate these association findings in a large case-control sample from Germany. Methods: The case group included 3657 patients with myocardial infarction and the control group comprised 1211 individuals with angiographically normal coronary arteries and without clinical signs or symptoms of myocardial infarction. Nine different polymorphisms were genotyped with the use of the TaqMan technique. Results: Genotype, allele, and haplotype analyses did not reveal significant associations between the polymorphisms and myocardial infarction. The negative results included a four-marker haplotype, termed HapA haplotype (odds ratio $=1.10 ; 95 \%$ confidence interval: $0.96-$ 1.25), that was previously found to be related with myocardial infarction in a sample from Iceland, and a different four-marker haplotype, termed HapB haplotype (odds ratio $=0.94 ; 95 \% \mathrm{Cl}$ : $0.79-1.12$ ), that was previously linked with myocardial infarction in a sample from the United Kingdom. Nine-marker haplotypes were not significantly associated with myocardial infarction in multiple logistic regression models adjusted for covariates ( $P \geq 0.38$ ). Conclusion: In this sample from central Europe, specific polymorphisms in the gene for 5-lipoxygenase-activating protein were not associated with myocardial infarction, a result contrasting previous positive findings. Genet Med 2007:9(2):123-129.
\end{abstract}

Key Words: ALOX5AP, 5-lipoxygenase-activating protein (FLAP), genetics, haplotype, myocardial infarction

Specific allelic forms of the gene encoding 5-lipoxygenaseactivating protein (FLAP) have been linked with susceptibility to myocardial infarction (MI) and stroke. ${ }^{1-4}$ These association findings may reflect a possible relationship of the regulatory function of FLAP in the inflammatory 5-lipoxygenase pathway and the important role attributed to inflammatory processes in atherosclerotic diseases. ${ }^{5-9}$ The 5 -lipoxygenase cascade leads to the formation of leukotrienes, which exhibit strong proinflammatory activities in cardiovascular tissues. ${ }^{9-11}$ This pathway is especially active in arterial walls of patients afflicted with various lesion stages of atherosclerosis of the aorta and of coronary and carotid arteries. ${ }^{10}$

From the ${ }^{I}$ Deutsches Herzzentrum München and 1. Medizinische Klinik, Klinikum rechts der Isar, Technische Universität München, Munich, Germany; ${ }^{2}$ Max Planck Institute for Ornithology, Department of Behavioural Ecology and Evolutionary Genetics, Starnberg (Seewiesen), Germany.

The authors declare no conflict of interest.

Werner Koch, PhD, German Heart Center Munich, Lazarettstrasse 36, 80636 München, Germany.E-mail: wkoch@dhm.mhn.de

A supplementary Appendix is available via the ArticlePlus feature at www.geneticsinmedicine. org. Please go to the February issue and click on the ArticlePlus link posted with the article in the Table of Contents to view this material.

Submitted for publication September 27, 2006.

Accepted for publication December 5, 2006.

DOI: 10.1097/GIM.0b013e318030c9c5
The gene for FLAP (ALOX5AP) contains five exons and spans approximately $31 \mathrm{~kb}$ in the chromosome $13 \mathrm{q} 12$ region. ${ }^{12,13}$ Specific single nucleotide polymorphisms (SNPs), named SG13S100, SG13S106, SG13S114, and a four-marker haplotype of ALOX5AP, termed HapA haplotype (SG13S25-G, SG13S114-T, SG13S89-G, SG13S32-A), were found to be related to $\mathrm{MI}$ in a population sample from Iceland. ${ }^{1} \mathrm{~A}$ different four-marker haplotype of $A L O X 5 A P$, termed HapB haplotype (SG13S377-A, SG13S114-A, SG13S41-A, SG13S35-G), but not the HapA haplotype, was linked with $\mathrm{MI}$ in a sample from the United Kingdom (UK). ${ }^{1}$ No evidence of an association of the HapA or HapB haplotype with MI was obtained in a sample of white male physicians from the United States (US). ${ }^{14}$

We examined whether the nine different SNPs mentioned above, nine-marker haplotypes of these SNPs, and the HapA and HapB haplotypes were associated with $\mathrm{MI}$ in a German population. The sample consisted of 3657 patients with MI and 1211 control individuals, all of whom were assessed with coronary angiography.

\section{METHODS}

\section{Patients and controls}

Participants were recruited from Southern Germany and examined at Deutsches Herzzentrum München or 1. Medizinische 
Klinik rechts der Isar der Technischen Universität München from 1993 to 2002. After catheterization, 5264 individuals were deemed eligible for inclusion in the MI or control group. Written informed consent for genetic analysis was obtained from $97.1 \%$ $(\mathrm{n}=5111)$ of these individuals. In no case was consent withdrawn. Blood samples assigned for DNA preparation had been collected from $95.2 \%(n=4868)$ of the individuals who agreed to participate in the study. These individuals, 3657 patients with MI and 1211 controls, constituted the study population. Complete genotype data were obtained from all these patients and control individuals. The study protocol was approved by the Institutional Ethics committee and the reported investigations were in accordance with the principles of the Declaration of Helsinki. ${ }^{15}$

\section{Definitions}

Individuals were considered disease free and, therefore, eligible as controls when their coronary arteries were angiographically normal and when they had no history of MI, no symptoms suggestive of MI, no electrocardiographic signs of MI, and no regional wall motion abnormalities. Coronary angiography in the control individuals was performed for the evaluation of chest pain. The diagnosis of MI was established in the presence of chest pain lasting longer than 20 minutes combined with ST-segment elevation or pathologic Q waves on a surface electrocardiogram. Patients with MI had to show either an angiographically occluded infarct-related artery or regional wall motion abnormalities corresponding to the electrocardiographic infarct localization, or both. Systemic arterial hypertension was defined as a systolic blood pressure of $\geq 140 \mathrm{~mm}$ $\mathrm{Hg}$ and/or a diastolic blood pressure of $\geq 90 \mathrm{~mm} \mathrm{Hg},{ }^{16}$ on at least two separate occasions or antihypertensive treatment. Hypercholesterolemia was defined as a documented total cholesterol value $\geq 240 \mathrm{mg} / \mathrm{dL}(\geq 6.2 \mathrm{mmol} / \mathrm{L})$ or current treatment with cholesterol-lowering medication. Persons reporting regular smoking in the previous 6 months were considered as current smokers. Diabetes mellitus was defined as the presence of an active treatment with insulin or an oral antidiabetic agent; for patients on dietary treatment, documentation of an abnormal fasting blood glucose or glucose tolerance test based on the World Health Organization criteria ${ }^{17}$ was required for establishing this diagnosis.

\section{Genetic analysis}

Genomic DNA was extracted from peripheral blood leukocytes with the QIAamp DNA Blood Kit (Qiagen, Hilden, Germany) or the High Pure PCR Template Preparation Kit (Roche Applied Science, Mannheim, Germany). We designed and used TaqMan allelic discrimination assays for genotype analysis of nine SNPs in ALOX5AP (Table 1). Primers and probes (Table 1) were synthesized by Applied Biosystems (Darmstadt,

Table 1

SNPs in the ALOX5AP genomic region and nucleotide sequences of primers and probes used in TaqMan reactions

\begin{tabular}{|c|c|c|c|c|c|c|}
\hline $\begin{array}{l}\text { deCODE } \\
\text { SNP ID }^{a}\end{array}$ & $\begin{array}{c}\text { NCBI } \\
\mathrm{dbSNP} \mathrm{ID}^{b}\end{array}$ & $\begin{array}{l}\text { SNP } \\
\text { bases }\end{array}$ & $\begin{array}{l}\text { Position in } \\
\text { AL512642 }\end{array}$ & Location & Primer $\left(5^{\prime} \rightarrow 3^{\prime}\right)$ & Probe $\left(5^{\prime} \rightarrow 3^{\prime}\right)^{d}$ \\
\hline SG13S25 & - & $\mathrm{G}>\mathrm{A}$ & 26663 & $\begin{array}{l}\text { Upstream of } \\
\text { exon } 1\end{array}$ & $\begin{array}{l}\text { TCTGACAGCATCAGCTAGTCTCTTTC } \\
\text { AAATTCATGTTGCTGTGTCCATACA }\end{array}$ & $\begin{array}{l}\text { FAM-CACTGTTGCCCAGTGG } \\
\text { VIC-AGCCACTGTTACCCAGT }\end{array}$ \\
\hline SG13S377 & - & $\mathrm{G}>\mathrm{A}$ & 31075 & $\begin{array}{l}\text { Upstream of } \\
\text { exon } 1\end{array}$ & $\begin{array}{l}\text { TTTGGCCAGACTGTCTTGAACTC } \\
\text { TGGCTCATGCCTATAATCACAAAA }\end{array}$ & $\begin{array}{l}\text { FAM-CCTGCCTCGGCCT } \\
\text { VIC-CTGCCTCAGGCTC }\end{array}$ \\
\hline SG13S100 & rs4073259 & $A>G$ & 33381 & $\begin{array}{l}\text { Upstream of } \\
\text { exon } 1\end{array}$ & $\begin{array}{l}\text { GGTGAAGTGGACTCCСTCCAT } \\
\text { CCCCGCTCTGAGCTCCTT }\end{array}$ & $\begin{array}{l}\text { FAM-AGCCAGCGCGCAG } \\
\text { VIC-CAGCCAGTGCGCAG }\end{array}$ \\
\hline SG13S106 & rs9579646 & $\mathrm{G}>\mathrm{A}$ & 37689 & Intron 1 & $\begin{array}{l}\text { TGTGTAGAGCTGTCTTCCTAAAGTTCTG } \\
\text { AAGCCACTGGAGATAGTTATGAAAGTG }\end{array}$ & $\begin{array}{l}\text { FAM-AGTTAGGGCTGCCTC } \\
\text { VIC-AGTTAGGACTGCCTCAG }\end{array}$ \\
\hline SG13S114 & rs10507391 & $\mathrm{T}>\mathrm{A}$ & 39206 & Intron 1 & $\begin{array}{l}\text { CCAGATGTATGTCCAAGCCTCTCT } \\
\text { CTCTGTAAGGTAGGTCTATGGTTGCAA }\end{array}$ & $\begin{array}{l}\text { FAM-TGCAATTCTAATTAACCTC } \\
\text { VIC-TGCAATTCTATTTAACCTC }\end{array}$ \\
\hline SG13S89 & rs4769874 & $\mathrm{G}>\mathrm{A}$ & 53551 & Intron 3 & $\begin{array}{l}\text { TCGGGAGGCCGTGTTTC } \\
\text { CCAGGGAGCAAGCATTAGCA }\end{array}$ & $\begin{array}{l}\text { FAM-ATTATCACACGCGCTCT } \\
\text { VIC-TATCACATGCGCTCTG }\end{array}$ \\
\hline SG13S32 & rs9551963 & $A>C$ & 59657 & Intron 4 & $\begin{array}{l}\text { CTGCTTTAGTTCTTGACCTCACCAA } \\
\text { CTGGGGTTCAAGAGAGAAATTCC }\end{array}$ & $\begin{array}{l}\text { FAM-AAGGATCTCATCTAGCAAT } \\
\text { VIC-AAGGATCTCATCGAGCAA }\end{array}$ \\
\hline SG13S41 & rs9315050 & $A>G$ & 63155 & Intron 4 & $\begin{array}{l}\text { CCTGTCTCCAAATACAGTCCCATT } \\
\text { AGGTCCCTTCCAAAATTCATATGTT }\end{array}$ & $\begin{array}{l}\text { FAM-ATCTTTACTCTCAGTTCCT } \\
\text { VIC-TCTTTACECTCAGTTCC }\end{array}$ \\
\hline SG13S35 & - & $\mathrm{G}>\mathrm{A}$ & 67227 & $\begin{array}{l}\text { Downstream } \\
\text { of exon } 5\end{array}$ & $\begin{array}{l}\text { CCTGGCATTGAGGAGTTTTCC } \\
\text { ACCCCACAAATACCTACAAATATGTGTAT }\end{array}$ & $\begin{array}{l}\text { FAM-TAAAAAACCGAAAGGAC } \\
\text { VIC-TTAAAAAACTGAAAGGACC }\end{array}$ \\
\hline \multicolumn{7}{|c|}{$\begin{array}{l}{ }^{a} \text { Helgadottir et al. }{ }^{1} \\
{ }^{b} \text { NCBI SNP database (http://www.ncbi.nlm.nih.gov/entrez/); last accessed September 27, } 2006 . \\
{ }^{c} \text { NCBI nucleotide database (http://www.ncbi.nlm.nih.gov/entrez/); sequence version of May 18, } 2005 . \\
{ }^{d} \text { FAM (6-carboxy-fluorescein) or VIC (proprietary dye of Applied Biosystems) was attached to the } 5^{\prime} \text { ends of the probe oligonucleotides. The sequences of the probes } \\
\text { used for analysis of the SG13S25, SG13S377, SG13S106, and SG13S114 SNPs corresponded to the coding strand and the sequences of the probes used for analysis of } \\
\text { the SG13S100, SG13S89, SG13S32, SG13S41, and SG13S35 SNPs corresponded to the noncoding strand; the allele-specific nucleotide in each probe sequence is } \\
\text { underlined. } \\
\text { SNPs, single nucleotide polymorphisms. }\end{array}$} \\
\hline
\end{tabular}


Table 2

Baseline characteristics of the control group and the MI group

\begin{tabular}{lccc}
\hline Characteristic & $\begin{array}{c}\text { Control group } \\
(n=1211)\end{array}$ & $\begin{array}{c}\text { MI group } \\
(n=3657)\end{array}$ & $P$ \\
\hline Age, yr & $60.3 \pm 11.9$ & $64.0 \pm 12.0$ & $<0.0001$ \\
Women & $598(49.4)$ & $885(24.2)$ & $<0.0001$ \\
Arterial hypertension & $589(48.6)$ & $2246(61.4)$ & $<0.0001$ \\
Hypercholesterolemia & $602(49.7)$ & $2067(56.5)$ & $<0.0001$ \\
Current cigarette smoking & $184(15.2)$ & $1849(50.6)$ & $<0.0001$ \\
Diabetes mellitus & $65(5.4)$ & $754(20.6)$ & $<0.0001$ \\
\hline
\end{tabular}

Age is mean $\pm \mathrm{SD}$; other variables are presented as number (\%).

MI, myocardial infarction.
Germany). To accomplish allele-specific signaling, the probes contained the fluorogenic dyes 6-carboxy-fluorescein (FAM) or VIC (proprietary dye of Applied Biosystems). Minor groove binder groups were conjugated with the $3^{\prime}$ ends of the oligonucleotides to facilitate formation of stable duplexes between the probes and their single-stranded DNA targets. ${ }^{18}$ Approximately $20 \%$ of the DNA samples were retyped with each TaqMan system to control for correct sample handling and data acquisition. The results of these repeat assays were in full agreement with the original genotyping results.

Analyses of PCR products with allele-discriminating restriction enzymes and/or DNA sequencing were used to verify the accuracy of TaqMan genotyping. We employed the restriction enzymes BglI (SG13S25 SNP), HaeIII (SG13S377 SNP), NsbI (SG13S100 SNP), SatI (SG13S106 SNP), TasI (SG13S114

Table 3

Genotype distributions and allele frequencies of ALOX5AP SNPs in the control group and the MI group

\begin{tabular}{|c|c|c|c|c|c|c|c|c|}
\hline deCODE SNP ID & Genotype & $\begin{array}{l}\text { Control group } \\
\text { (1211 genotypes) }\end{array}$ & $\begin{array}{c}\text { MI group } \\
\text { (3657 genotypes) }\end{array}$ & $P$ & Allele & $\begin{array}{l}\text { Control group } \\
\text { (2422 alleles) }\end{array}$ & $\begin{array}{c}\text { MI group } \\
\text { (7314 alleles) }\end{array}$ & $P$ \\
\hline \multirow[t]{3}{*}{ SG13S25 } & GG & $963(79.5)$ & $2949(80.6)$ & 0.54 & G & $2162(89.3)$ & $6579(90.0)$ & 0.33 \\
\hline & GA & $236(19.5)$ & $681(18.6)$ & & A & $260(10.7)$ & $735(10.0)$ & \\
\hline & AA & $12(1.0)$ & $27(0.7)$ & & & & & \\
\hline \multirow[t]{3}{*}{ SG13S377 } & GG & $861(71.1)$ & $2714(74.2)$ & 0.053 & G & $2047(84.5)$ & $6285(85.9)$ & 0.086 \\
\hline & GA & $325(26.8)$ & $857(23.4)$ & & A & 375 (15.5) & $1029(14.1)$ & \\
\hline & AA & $25(2.1)$ & $86(2.4)$ & & & & & \\
\hline \multirow[t]{3}{*}{ SG13S100 } & AA & $494(40.8)$ & $1461(40.0)$ & 0.11 & A & $1521(62.8)$ & $4636(63.4)$ & 0.60 \\
\hline & AG & $533(44.0)$ & $1714(46.9)$ & & G & $901(37.2)$ & $2678(36.6)$ & \\
\hline & GG & $184(15.2)$ & $482(13.2)$ & & & & & \\
\hline \multirow[t]{3}{*}{ SG13S106 } & GG & $568(46.9)$ & $1697(46.4)$ & 0.27 & G & $1644(67.9)$ & $4998(68.3)$ & 0.68 \\
\hline & GA & 508 (41.9) & 1604 (43.9) & & A & $778(32.1)$ & $2316(31.7)$ & \\
\hline & AA & $135(11.1)$ & $356(9.7)$ & & & & & \\
\hline \multirow[t]{3}{*}{ SG13S114 } & $\mathrm{TT}$ & $526(43.4)$ & $1591(43.5)$ & 0.40 & $\mathrm{~T}$ & $1586(65.5)$ & $4842(66.2)$ & 0.52 \\
\hline & TA & $534(44.1)$ & $1660(45.4)$ & & A & $836(34.5)$ & $2472(33.8)$ & \\
\hline & AA & $151(12.5)$ & $406(11.1)$ & & & & & \\
\hline \multirow[t]{3}{*}{ SG13S89 } & GG & $1093(90.3)$ & $3332(91.1)$ & 0.60 & G & $2301(95.0)$ & 6983 (95.5) & 0.34 \\
\hline & GA & $115(9.5)$ & $319(8.7)$ & & A & $121(5.0)$ & $331(4.5)$ & \\
\hline & AA & $3(0.2)$ & $6(0.2)$ & & & & & \\
\hline \multirow[t]{3}{*}{ SG13S32 } & AA & 301 (24.9) & $924(25.3)$ & 0.39 & A & $1224(50.5)$ & 3650 (49.9) & 0.59 \\
\hline & $\mathrm{AC}$ & $622(51.4)$ & $1802(49.3)$ & & $\mathrm{C}$ & $1198(49.5)$ & $3664(50.1)$ & \\
\hline & $\mathrm{CC}$ & $288(23.8)$ & $931(25.5)$ & & & & & \\
\hline \multirow[t]{3}{*}{ SG13S41 } & AA & $1047(86.5)$ & $3166(86.6)$ & 0.96 & A & $2253(93.0)$ & $6810(93.1)$ & 0.88 \\
\hline & AG & $159(13.1)$ & $478(13.1)$ & & G & $169(7.0)$ & $504(6.9)$ & \\
\hline & GG & $5(0.4)$ & $13(0.4)$ & & & & & \\
\hline \multirow[t]{3}{*}{ SG13S35 } & GG & $977(80.7)$ & 3025 (82.7) & 0.24 & G & $2172(89.7)$ & 6645 (90.9) & 0.086 \\
\hline & GA & $218(18.0)$ & $595(16.3)$ & & A & $250(10.3)$ & $669(9.1)$ & \\
\hline & AA & $16(1.3)$ & $37(1.0)$ & & & & & \\
\hline
\end{tabular}

Variables are presented as number (\%) of genotypes or alleles in control individuals and myocardial infarction patients.

SNPs, single nucleotide polymorphisms. 
SNP), XceI (SG13S89 SNP), TaqI (SG13S32 SNP), and BslLI (SG13S41 SNP) (MBI Fermentas). DNA sequencing was used to test whether one or more additional polymorphisms were present in the probe-binding section of the amplicons, because they may interfere with TaqMan reactions and result in wrong genotype assignments. With each SNP, 100 DNA samples were examined by sequencing. The known SNPs were identified as the only sequence variabilities in the probe-binding regions. Thus the probability of genotyping errors due to possible further sequence variations was relatively low.

Clinicians responsible for diagnosis were not aware of the genetic data. All genetic analyses were blinded.

\section{Statistical analysis}

The analysis consisted of comparisons of genotype, allele, and haplotype frequencies between the control group and the group of patients with MI. Because stronger associations of the HapA haplotype with MI were observed in men compared to women in both the Iceland and UK studies, ${ }^{1}$ we also conducted separate analyses of SNP genotype distributions and HapA and HapB haplotype frequencies in the groups of men and women. Discrete variables are expressed as counts (\%) and compared using the $\chi^{2}$ test. Continuous variables are expressed as mean $\pm \mathrm{SD}$ and compared by means of the unpaired, two-sided $t$ test. Haplotypes were reconstructed from genotype data with the use of the software

Table 4

Genotype distributions of ALOX5AP SNPs in the women and men of the control group and the MI group

\begin{tabular}{|c|c|c|c|c|c|c|c|}
\hline \multirow[b]{2}{*}{ deCODE SNP ID } & \multirow[b]{2}{*}{ Genotype } & \multicolumn{3}{|c|}{ Women } & \multicolumn{3}{|c|}{ Men } \\
\hline & & $\begin{array}{l}\text { Control group } \\
(\mathrm{n}=598)\end{array}$ & $\begin{array}{l}\text { MI group } \\
(\mathrm{n}=885)\end{array}$ & $P$ & $\begin{array}{l}\text { Control group } \\
\quad(\mathrm{n}=613)\end{array}$ & $\begin{array}{l}\text { MI group } \\
(\mathrm{n}=2772)\end{array}$ & $P$ \\
\hline \multirow[t]{3}{*}{ SG13S25 } & GG & $465(77.8 \%)$ & $716(80.9 \%)$ & 0.13 & $498(81.2 \%)$ & $2233(80.6 \%)$ & 0.87 \\
\hline & GA & $127(21.2 \%)$ & $166(18.8 \%)$ & & $109(17.8 \%)$ & $515(18.6 \%)$ & \\
\hline & AA & $6(1.0 \%)$ & $3(0.3 \%)$ & & $6(1.0 \%)$ & $24(0.9 \%)$ & \\
\hline \multirow[t]{3}{*}{ SG13S377 } & GG & $429(71.7 \%)$ & $659(74.5 \%)$ & 0.37 & $432(70.5 \%)$ & $2055(74.1 \%)$ & 0.13 \\
\hline & GA & $157(26.3 \%)$ & $205(23.2 \%)$ & & $168(27.4 \%)$ & $652(23.5 \%)$ & \\
\hline & AA & $12(2.0 \%)$ & $21(2.4 \%)$ & & $13(2.1 \%)$ & $65(2.3 \%)$ & \\
\hline \multirow[t]{3}{*}{ SG13S100 } & AA & $255(42.6 \%)$ & $372(42.0 \%)$ & 0.64 & $239(39.0 \%)$ & $1089(39.3 \%)$ & 0.10 \\
\hline & AG & $261(43.6 \%)$ & $404(45.6 \%)$ & & $272(44.4 \%)$ & $1310(47.3 \%)$ & \\
\hline & GG & $82(13.7 \%)$ & $109(12.3 \%)$ & & $102(16.6 \%)$ & $373(13.5 \%)$ & \\
\hline \multirow[t]{3}{*}{ SG13S106 } & GG & $291(48.7 \%)$ & $431(48.7 \%)$ & 0.66 & $277(45.2 \%)$ & $1266(45.7 \%)$ & 0.27 \\
\hline & GA & $247(41.3 \%)$ & $377(42.6 \%)$ & & $261(42.6 \%)$ & $1227(44.3 \%)$ & \\
\hline & AA & $60(10.0 \%)$ & $77(8.7 \%)$ & & $75(12.2 \%)$ & $279(10.1 \%)$ & \\
\hline \multirow[t]{3}{*}{ SG13S114 } & TT & $270(45.2 \%)$ & $403(45.5 \%)$ & 0.65 & $256(41.8 \%)$ & $1188(42.9 \%)$ & 0.53 \\
\hline & TA & $256(42.8 \%)$ & $389(44.0 \%)$ & & $278(45.4 \%)$ & $1271(45.9 \%)$ & \\
\hline & AA & $72(12.0 \%)$ & $93(10.5 \%)$ & & $79(12.9 \%)$ & $313(11.3 \%)$ & \\
\hline \multirow[t]{3}{*}{ SG13S89 } & GG & $545(91.1 \%)$ & $813(91.9 \%)$ & 0.84 & $548(89.4 \%)$ & $2519(90.9 \%)$ & 0.37 \\
\hline & GA & $52(8.7 \%)$ & $70(7.9 \%)$ & & $63(10.3 \%)$ & $249(9.0 \%)$ & \\
\hline & AA & $1(0.2 \%)$ & $2(0.2 \%)$ & & $2(0.3 \%)$ & $4(0.1 \%)$ & \\
\hline \multirow[t]{3}{*}{ SG13S32 } & AA & $147(24.6 \%)$ & $221(25.0 \%)$ & 0.51 & $154(25.1 \%)$ & $703(25.4 \%)$ & 0.89 \\
\hline & $\mathrm{AC}$ & $315(52.7 \%)$ & $442(49.9 \%)$ & & $307(50.1 \%)$ & $1360(49.1 \%)$ & \\
\hline & $\mathrm{CC}$ & $136(22.7 \%)$ & $222(25.1 \%)$ & & $152(24.8 \%)$ & $709(25.6 \%)$ & \\
\hline \multirow[t]{3}{*}{ SG13S41 } & AA & $524(87.6 \%)$ & $773(87.3 \%)$ & 0.99 & $523(85.3 \%)$ & $2393(86.3 \%)$ & 0.75 \\
\hline & AG & $72(12.0 \%)$ & $109(12.3 \%)$ & & $87(14.2 \%)$ & $369(13.3 \%)$ & \\
\hline & GG & $2(0.3 \%)$ & $3(0.3 \%)$ & & $3(0.5 \%)$ & $10(0.4 \%)$ & \\
\hline \multirow[t]{3}{*}{ SG13S35 } & GG & $472(78.9 \%)$ & $722(81.6 \%)$ & 0.19 & $505(82.4 \%)$ & $2303(83.1 \%)$ & 0.59 \\
\hline & GA & $114(19.1 \%)$ & $154(17.4 \%)$ & & $104(17.0 \%)$ & $441(15.9 \%)$ & \\
\hline & AA & $12(2.0 \%)$ & $9(1.0 \%)$ & & $4(0.7 \%)$ & $28(1.0 \%)$ & \\
\hline
\end{tabular}

Variables are presented as number (\%) of genotypes in control individuals and MI patients.

SNPs, single nucleotide polymorphisms; MI, myocardial infarction. 
package PHASE. ${ }^{19}$ We tested for the independent association effect of nine-marker haplotypes in multiple logistic regression models of MI that included as covariates age, gender, history of arterial hypertension, history of hypercholesterolemia, current cigarette smoking, and diabetes mellitus. Adjusted odds ratios and 95\% Wald confidence intervals were calculated based on these models. Statistical significance was set at $P<0.05$.

\section{RESULTS}

The main baseline characteristics of the control group $(n=1211)$ and the group of patients with MI $(\mathrm{n}=3657)$ are shown in Table 2. Mean age of the MI patients was higher than that of the control group; the proportion of women was lower in the patient group than in the control group; and history of arterial hypertension and hypercholesterolemia, current cigarette smoking, and diabetes mellitus were more prevalent in the MI patient group than in the control group ( $P<0.0001$ for all comparisons; Table 2$)$.

Genotype distributions and allele frequencies of the ALOX5AP SNPs were not significantly different between the control group and patient group (Table 3). Significant sex-related differences of the genotype distributions were not found (Table 4).

Figure 1 shows the linkage disequilibrium (LD) block structure defined by the nine genotyped SNPs. Strong LD was

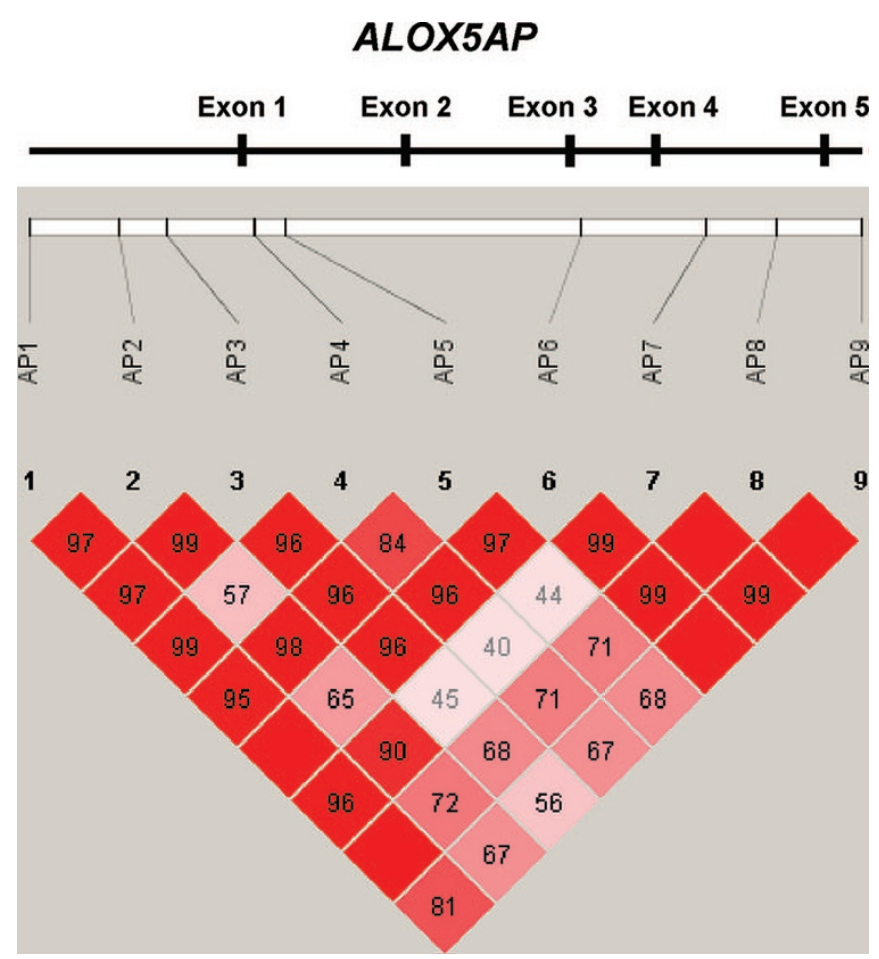

Fig. 1. Genetic diversity at the ALOX5AP genomic region located in the long arm of chromosome 13 (band q12). The exon-intron structure was adapted from sequence data deposited in the NCBI nucleotide database (http://www.ncbi.nlm.nih.gov/entrez/) under accession number AL512642, version of May 18, 2005. The values within squares show the pairwise correlations between single nucleotide polymorphisms (SNPs) (measured as $D^{\prime}$ ) defined at the top left and top right sides of the squares. Squares without a number indicate $D^{\prime}=1.00$. SNP designations: $\mathrm{AP} 1=\mathrm{SG} 13 \mathrm{~S} 25, \mathrm{AP} 2=\mathrm{SG} 13 \mathrm{~S} 377, \mathrm{AP} 3=$ SG13S100, AP4 = SG13S106, AP5 = SG13S114, AP6 = SG13S89, AP7 = SG13S32, AP8 = SG13S41, AP9 $=$ SG13S35.
Table 5

Frequencies of the HapA and HapB haplotypes in the control and MI groups

\begin{tabular}{lccc}
\hline Haplotype & $\begin{array}{c}\text { Control group } \\
(2422 \text { haplotypes })\end{array}$ & $\begin{array}{c}\text { MI group } \\
\text { (7314 haplotypes })\end{array}$ & $P$ \\
\hline HapA & $359(14.8)$ & $1171(16.0)$ & 0.16 \\
HapB & $182(7.5)$ & $518(7.1)$ & 0.48 \\
\hline
\end{tabular}

Haplotype frequencies are presented as number (\%). The HapA haplotype is defined by the alleles SG13S25-G, SG13S114-T, SG13S89-G, and SG13S32-A, and the HapB haplotype is defined by the alleles SG13S377-A, SG13S114-A, SG13S41-A, and SG13S35-G (Helgadottir et al. ${ }^{1}$ ).

MI, myocardial infarction.

present across the ALOX5AP region (Fig. 1). Frequencies of the HapA (SG13S25-G, SG13S114-T, SG13S89-G, SG13S32-A) and HapB (SG13S377-A, SG13S114-A, SG13S41-A, SG13S35-G) haplotypes were not substantially different between the control group and the patient group (Table 5). Risk estimates were 1.10 (95\% CI: 0.96-1.25) for the HapA haplotype and 0.94 (95\% CI: $0.79-1.12$ ) for the HapB haplotype. Haplotypes defined by nine SNPs were not present at significantly different proportions among the control individuals and patients, with the exception of the Hap5 haplotype, which showed a moderately higher frequency in the control group than in the patient group (Table 6).

The frequencies of the HapA, HapB, and nine-marker haplotypes were not significantly different between the women of the control and MI groups and between the men of the two groups. In addition, we did not observe significant differences in age or sex between the carriers and noncarriers of specific haplotypes in the control group.

To assess whether independent associations existed between nine-marker haplotypes and MI, we performed a multivariate logistic regression analysis. After adjustments were made for conventional cardiovascular risk markers (age, gender, history of arterial hypertension, history of hypercholesterolemia, cur-

Table 6

Frequencies of nine-marker haplotypes in the control and MI groups

\begin{tabular}{|c|c|c|c|c|}
\hline & Haplotype & & & \\
\hline Name & Allele combination & (2422 haplotypes) & (7314 haplotypes) & $P$ \\
\hline Hap1 & GGAGTGCAG & $928(38.3)$ & $2805(38.4)$ & 0.98 \\
\hline Hap2 & GGAGTGAAG & $237(9.8)$ & $808(11.0)$ & 0.082 \\
\hline Hap3 & AGAGTGAAG & $253(10.4)$ & $705(9.6)$ & 0.25 \\
\hline Hap4 & GGGAAGAAG & $204(8.4)$ & $665(9.1)$ & 0.32 \\
\hline Hap5 & GAGAAGAAA & $188(7.8)$ & $479(6.5)$ & 0.041 \\
\hline & Other & $612(25.3)$ & $1852(25.3)$ & 0.96 \\
\hline
\end{tabular}

Haplotype frequencies are presented as number (\%). Shown are results obtained from the five most frequent nine-marker haplotypes and the combined other nine-marker haplotypes. Each haplotype is defined as a specific allele combination based on nine single nucleotide polymorphisms (SNPs) in ALOX5AP. The order of the SNPs is as follows (from left to right): SG13S25, SG13S377, SG13S100, SG13S106, SG13S114, SG13S89, SG13S32, SG13S41, SG13S35. Overall $P=0.12$. See Table 1 and Figure 1 for the locations of the SNPs in the ALOX5AP genomic region.

$\mathrm{MI}$, myocardial infarction. 
rent cigarette smoking, diabetes mellitus), the analysis showed that none of the five most frequent nine-marker haplotypes, including the Hap5 haplotype, or the combined other haplotypes were significantly related with MI $(P \geq 0.38)$.

\section{DISCUSSION}

The present data show that specific SNPs in ALOX5AP are not associated with $\mathrm{MI}$ in a large German population. Analyses in women and men did not reveal sex-specific relationships between the SNPs and MI. Specific four-marker haplotypes, the HapA and HapB haplotypes, and nine-marker haplotypes were not associated with MI. Most SNPs were in strong LD and the LD block structure was similar to those in other white populations. ${ }^{4,14}$ Three of the nine SNPs examined here, the SG13S100, SG13S106, and SG13S114, were found to be significantly associated with $\mathrm{MI}$ in a population from Iceland that consisted of 779 unrelated patients with MI and 624 population-based control individuals. ${ }^{1}$ However, significant associations between these SNPs and MI were not observed when adjustments were made for the number of markers tested. ${ }^{1}$ None of the three SNPs was associated with MI in the present population. The HapA haplotype was associated with a twofold greater risk of MI in the Icelandic population (nominal $P=0.0000023$; adjusted $P=0.005$ ) but not in a sample from the UK (753 patients with MI and 730 control individuals). ${ }^{1}$ In the same UK population, the HapB haplotype was associated with MI (nominal $P=0.00037$; adjusted $P=0.046$ ). ${ }^{1}$

The control subjects had some indication for coronary angiography, and, therefore, they did not constitute a typical sample of healthy controls. We compared the frequencies of the HapA haplotype and the SNP alleles that define the HapA haplotype between the present control sample and an independent control group that consisted of 736 unrelated individuals from the KORAS2000 sample, a representative local population sample from southern Germany. ${ }^{4}$ In the present control group and the control group from the KORAS2000 sample, ${ }^{4}$ the frequencies of the HapA haplotype were $14.8 \%$ versus $15.2 \%(P=0.74)$ and the frequencies of the SG13S25-G, SG13S114-T, SG13S89-G, and SG13S32-A alleles were 89.3\% vs. $90.1 \%(P=0.42), 65.5 \%$ vs. $65.0 \%(P=0.77), 95.0 \%$ vs. $96.0 \%(P=0.15)$, and $50.5 \%$ vs. $49.7 \%(P=0.62)$, respec- tively. Thus, with regard to the frequencies of the HapA haplotype and the alleles that constitute the HapA haplotype, the present control group is not substantially different from an established population-based sample. We inferred from this finding that the control sample with coronary angiography was suitable for the genetic association study described here. Measures of inflammation were not examined, which is a limitation of the current study.

Relationships of ALOX5AP SNPs and haplotypes with MI and ischemic stroke were evaluated in a nested case-control study within the Physicians' Health Study cohort that comprised predominantly white (>94\%) male US physicians. ${ }^{14,20}$ Investigation of $341 \mathrm{MI}$ case-control pairs did not provide evidence of an association of any of the tested SNPs or the HapA or HapB haplotype with MI. ${ }^{14}$ Genotype distributions and frequencies of SNP alleles and the HapA and HapB haplotypes in the case and control groups of the US sample ${ }^{14}$ corresponded well with those of the present German sample.

Similar to results obtained in Germans (this study) and US physicians, ${ }^{14}$ the SNPs that define the HapA and HapB haplotypes were not associated with $\mathrm{MI}$ in a Japanese population that included 353 patients with MI and 1875 control individuals. ${ }^{2}$ A meaningful association analysis of the HapA and HapB haplotypes was not possible in the sample from Japan because, with some of the SNPs, minor alleles were either absent or extremely rare. ${ }^{2}$ Two-marker ALOX5AP haplotypes not related to the HapA and HapB haplotypes were associated with MI in the Japanese sample. ${ }^{2}$

Studies conducted with samples of white individuals provided heterogeneous results about the relationship of the HapA and HapB haplotypes with MI (Table 7). Association of the HapA haplotype with MI was observed in a study sample from Iceland, but this finding was not replicated in samples from Germany (present study), the UK, and the US. ${ }^{1,14}$ A relationship of the HapB haplotype with MI was found in a study sample from the UK, but this result was not confirmed in samples from Germany (present study) and the US. ${ }^{1,14}$ Heterogeneities of genetic and environmental factors across the source populations are unlikely to account for the inconsistencies. Genetic markers for proposed gene-disease associations may vary in frequency between populations, but there is empirical evidence that their biological impact on the risk of common diseases is

Table 7

Frequencies of the HapA and Hap B haplotypes and estimated risks of MI associated with these haplotypes in case-control studies conducted with white population samples

\begin{tabular}{|c|c|c|c|c|c|c|}
\hline \multirow[b]{2}{*}{ Study } & \multicolumn{3}{|c|}{ HapA } & \multicolumn{3}{|c|}{ НарВ } \\
\hline & Controls/cases & Risk & $P$ & Controls/cases & Risk & $P$ \\
\hline Germany (present) & $0.15 / 0.16$ & 1.10 & 0.16 & $0.08 / 0.07$ & 0.94 & 0.48 \\
\hline United States ${ }^{14}$ & $0.14 / 0.17$ & 1.18 & 0.46 & $0.07 / 0.06$ & 0.62 & 0.08 \\
\hline Iceland ${ }^{1}$ & $0.10 / 0.16$ & 1.80 & $<0.005^{a}$ & \multicolumn{3}{|c|}{ No data available } \\
\hline United Kingdom ${ }^{1}$ & $0.15 / 0.17$ & n.s. & & $0.04 / 0.08$ & 1.95 & $0.046^{a}$ \\
\hline
\end{tabular}

Haplotype frequencies are presented as proportions of controls and cases; n.s. not significant (data not shown). ${ }^{1}$

${ }^{a}$ Adjusted for the number of haplotypes tested. ${ }^{1}$ 
usually consistent even across ethnic boundaries. ${ }^{21}$ Consistent replication of genetic associations has been difficult to achieve, despite the biological plausibility of these associations. ${ }^{22}$ In this context, the present findings argue against association of defined SNPs and haplotypes of ALOX5AP with MI.

\section{ACKNOWLEDGMENTS}

The work was entirely supported by institutional financing from the German Heart Center Munich. The authors thank Wolfgang Latz, Marianne Eichinger, and Claudia Ganser for excellent technical assistance.

\section{References}

1. Helgadottir A, Manolescu A, Thorleifsson G, Gretarsdottir S, et al. The gene encoding 5-lipoxygenase activating protein confers risk of myocardial infarction and stroke. Nat Genet 2004;36:233-239.

2. Kajimoto K, Shioji K, Ishida C, Iwanaga Y, et al. Validation of the association between the gene encoding 5-lipoxygenase-activating protein and myocardial infarction in a Japanese population. Circ J 2005;69:1029-1034.

3. Helgadottir A, Gretarsdottir S, St. Clair D, Manolescu A, et al. Association between the gene encoding 5-lipoxygenase-activating protein and stroke replicated in a Scottish population. Am J Hum Genet 2005;76:505-509.

4. Lõhmussaar E, Gschwendtner A, Mueller JC, Org T, et al. ALOX5AP gene and the PDE4D gene in a Central European population of stroke patients. Stroke 2005;36: 731-736.

5. Miller DK, Gillard JW, Vickers PJ, Sadowski S, et al. Identification and isolation of a membrane protein necessary for leukotriene production. Nature 1990;343:278-281.

6. Dixon RAF, Diehl RE, Opas E, Rands E, et al. Requirement of a 5-lipoxygenaseactivating protein for leukotriene synthesis. Nature 1990;343:282-284.

7. Libby P, Ridker PM, Maseri A. Inflammation and atherosclerosis. Circulation 2002; 105:1135-1143.

8. Dwyer JH, Allayee H, Dwyer KM, Fan J, et al. Arachidonate 5-lipoxygenase promoter genotype, dietary arachidonic acid, and atherosclerosis. N Engl J Med 2004; 350:29-37.
9. De Caterina R, Zampolli A. From asthma to atherosclerosis - 5-lipoxygenase, leukotrienes, and inflammation. N Engl J Med 2004;350:4-7.

10. Spanbroek R, Gräbner R, Lötzer K, Hildner M, et al. Expanding expression of the 5-lipoxygenase pathway within the arterial wall during human atherogenesis. Proc Natl Acad Sci U S A 2003;100:1238-1243.

11. Zhao L, Funk CD. Lipoxygenase pathways in atherogenesis. Trends Cardiovasc Med 2004;14:191-195.

12. Kennedy BP, Diehl RE, Boie Y, Adam M, et al. Gene characterization and promoter analysis of the human 5-lipoxygenase-activating protein (FLAP). J Biol Chem 1991; 266:8511-8516.

13. Yandava CN, Kennedy BP, Pillari A, Duncan AM, et al. Cytogenetic and radiation hybrid mapping of human arachidonate 5-lipoxygenase-activating protein (ALOX5AP) to chromosome 13q12. Genomics 1999;56:131-133.

14. Zee RYL, Cheng S, Hegener HH, Erlich HA, et al. Genetic variants of arachidonate 5-lipoxygenase-activating protein, and risk of incident myocardial infarction and ischemic stroke. A nested case-control approach. Stroke 2006;37:20072011.

15. World Medical Association declaration of Helsinki. Recommendations guiding physicians in biomedical research involving human subjects. JAMA 1997;277:925926.

16. Chalmers J, MacMahon S, Mancia G, Whitworth J, et al. 1999 World Health Organization-International Society of Hypertension Guidelines for the management of hypertension. Guidelines sub-committee of the World Health Organization. Clin Exp Hypertens 1999;21:1009-1060.

17. Diabetes mellitus. World Health Organization Study Group. Diabetes mellitus. WHO Tech Rep Ser 1985;727:1-104.

18. Kutyavin IV, Afonina IA, Mills A, Gorn VV, et al. 3'-Minor groove binder-DNA probes increase sequence specificity at PCR extension temperatures. Nucleic Acids Res 2000;28:655-661.

19. Stephens M, Smith NJ, Donnelly P. A new statistical method for haplotype reconstruction from population data. Am J Hum Genet 2001;68:978-989.

20. Final report on the aspirin component of the ongoing Physicians' Health Study. Steering committee of the Physicians' Health Study research group. N Engl J Med 1989;321:129-135.

21. Ioannidis JPA, Ntzani EE, Trikalinos TA. 'Racial' differences in genetic effects for complex diseases. Nat Genet 2004;36:1312-1318.

22. Hirschhorn JN, Lohmueller K, Byrne E, Hirschhorn K. A comprehensive review of genetic association studies. Genet Med 2002;4:45-61. 